
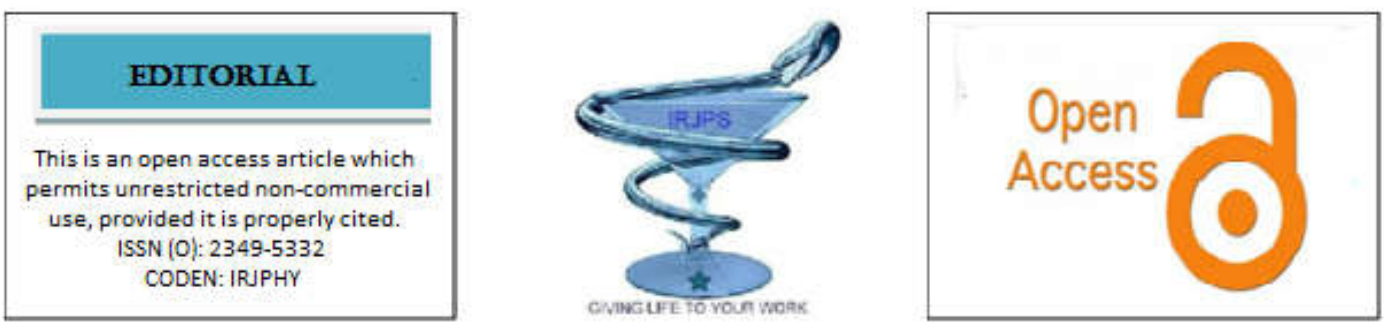

EDITORIAL

\title{
GENERAL STRESS SYNDROME AND POSSIBLE THERAPEUTIC INTERFERENCES
}

In this editorial, I am going to introduce a syndrome I am calling it as general stress syndrome (GSS). We are always exposed to stress from multiple sources, including biological, chemical and physical sources in broad terms. Long exposure to stress will end in a somatic disease. Now, most diseases may due to stress including diabetes, hypertension, neurologic diseases, cancer, autoimmune diseases,. etc. Therapeutic options usually are initiated for treating the existing episodes of a disease rather than its real roots. Take diabetes as an example, therapeutic options are based on giving metformin and ending with insulin. No real options are planned to treat stress as a causing agent of diabetes. The therapeutic options for other diseases do not involve deep insights of stress.

As time passes, two phenomena are more likely to occur, the first is the exposure to more stressors, and the second is the inability to tolerate more stress. The results may be clinically obvious through continuous feeling of fatigue, irritability for mild issues.

If we agreed that about this syndrome, GSS, as described, then GSS has to be seriously taken into account as therapeutic strategies may take other paths

\section{Ahed J Alkhatib}

Department of Legal Medicine, Toxicology and Forensic Medicine, Faculty of Medicine, Jordan University of Science and Technology, Jordan.

International Mariinskaya Academy, Department of medicine and critical care, Academician secretary of department of Sociology
Indian Research Journal of Pharmacy and Science; 24(2020)2041;

Journal Home Page: https://www.irjps.in DOI: 10.21276/irjps.2020.7.1.1 